\title{
The Fourier-Kelvin Stellar Interferometer: a practical interferometer for the detection and characterization of extrasolar giant planets
}

William C. Danchi, Ronald J. Allen, Dominic J. Benford, Drake Deming, Daniel Y. Gezari, et al.

William C. Danchi, Ronald J. Allen, Dominic J. Benford, Drake Deming, Daniel Y. Gezari, Marc J. Kuchner, David T. Leisawitz, Roger P. Linfield, Rafael Millan-Gabet, John D. Monnier, Lee G. Mundy, Charley Noecker, Jayadev K. Rajagopal, L. J. Richardson, Stephen A. Rinehart, Sara Seager, Wesley Arthur Traub, Debra J. Wallace, "The Fourier-Kelvin Stellar Interferometer: a practical interferometer for the detection and characterization of extrasolar giant planets," Proc. SPIE 5491, New Frontiers in Stellar Interferometry, (20 October 2004); doi: 10.1117/12.552077

Event: SPIE Astronomical Telescopes + Instrumentation, 2004, Glasgow, United Kingdom 


\title{
THE FOURIER-KELVIN STELLAR INTERFEROMETER: A practical interferometer for the detection and characterization of extrasolar giant planets
}

\author{
W. C. Danchi ${ }^{a}$, R. J. Allen ${ }^{\mathrm{b}}$, D.J. Benford ${ }^{\mathrm{a}}$, D. Deming ${ }^{\mathrm{a}}$, D. Y. Gezari ${ }^{\mathrm{a}}$, M. Kuchner ${ }^{\mathrm{c}}$, \\ D. T. Leisawitz ${ }^{\mathrm{a}}$, R. Linfield ${ }^{\mathrm{d}}$, R. Millan-Gabet ${ }^{\mathrm{e}}$, J. D. Monnier $^{\mathrm{f}}$, L. G. Mundy ${ }^{\mathrm{g}}$, C. Noecker ${ }^{\mathrm{d}}$, \\ J. Rajagopal ${ }^{\text {a,g }}$, L. J. Richardson ${ }^{\mathrm{a}}$. S. Rinehart ${ }^{\mathrm{a}}$, S. Seager ${ }^{\mathrm{h}}$, W. A. Traub ${ }^{\mathrm{c}}$. D. Wallace ${ }^{\mathrm{a}}$ \\ ${ }^{a}$ NASA Goddard Space Flight Center, Greenbelt, MD 20771, USA \\ ${ }^{\mathrm{b}}$ Space Telescope Science Institute,3700 San Martin Dr., Baltimore, MD 21218, USA \\ 'Harvard-Smithsonian Center for Astrophysics, 60 Garden St., Cambridge, MA 02138, USA \\ ${ }^{\mathrm{d}}$ Ball Aerospace, Boulder, 1600 Commerce St., CO 80301, USA \\ ${ }^{\mathrm{e}}$ California Institute of Technology, 1201 E. California Blvd., Pasadena, CA 91125, USA \\ fUniversity of Michigan, Astronomy Department, Ann Arbor, MI 48109, USA \\ ${ }^{\mathrm{g}}$ University of Maryland, Astronomy Department, College Park, MD 20742, USA \\ ${ }^{\mathrm{h}}$ Carnegie Institution of Washington,5241 Broad Branch Rd., NW, Washington, DC 20015, USA
}

\begin{abstract}
The Fourier-Kelvin Stellar Interferometer (FKSI) is a mission concept for a nulling interferometer for the near-tomid-infrared spectral region $(3-8 \mu \mathrm{m})$. FKSI is conceived as a scientific and technological precursor to TPF. The scientific emphasis of the mission is on the evolution of protostellar systems, from just after the collapse of the precursor molecular cloud core, through the formation of the disk surrounding the protostar, the formation of planets in the disk, and eventual dispersal of the disk material. FKSI will answer key questions about extrasolar planets:

- What are the characteristics of the known extrasolar giant planets?

- What are the characteristics of the extrasolar zodiacal clouds around nearby stars?

- Are there giant planets around classes of stars other than those already studied?

We present preliminary results of a detailed design study of the FKSI. Using a nulling interferometer configuration, the optical system consists of two $0.5 \mathrm{~m}$ telescopes on a $12.5 \mathrm{~m}$ boom feeding a Mach-Zender beam combiner with a fiber wavefront error reducer to produce a $0.01 \%$ null of the central starlight. With this system, planets around nearby stars can be detected and characterized using a combination of spectral and spatial resolution.
\end{abstract}

Keywords: mid-infrared, nulling interferometer, extrasolar planets, giant planets, space interferometer

\section{INTRODUCTION}

At the present time, more than 100 extra-solar planets have been found using high-precision Doppler velocity searches [1]. Considerable information exists about the masses of the planets (but with the sin $i$ ambiguity), the orbital periods and eccentricities. In a few cases planetary systems have been found [1]. Recently, sodium absorption in the atmospheric spectrum of the transiting planet has been measured. [2]. However, little else is known about these planets. Basic questions of great scientific importance include the density of the planets, i.e., whether they are rocky or gas giants (although most are expected to be gas giants), and the constituents and physical conditions of their atmospheres. Some further progress can be expected from ground-based astronomy, namely, nulling [3], and differential phase [4] experiments with the Keck Interferometer [5].

However, a dedicated interferometer located on a satellite platform provides the greatest long-term potential for progress on extra-solar planet research at infrared wavelengths, which eventually will lead to the ambitious and technically difficult Terrestrial Planet Finder (TPF) [6] and Darwin missions [7] for the detection and characterization of earth-like planets around the habitable zone [8]. 
Our team has been studying various interferometer architectures and beam combination techniques, and evaluating the relevant science and technology tradeoffs in preparation for future proposal opportunities for the FKSI mission. Some of the technical challenges include the development of the cryocooler systems necessary for the telescopes and focal plane array, light and stiff but well-damped truss systems to support the telescopes, and lightweight and coolable optical telescopes.

We present major findings of detailed design studies of the FKSI beginning with a design consisting of five one meter diameter telescopes arranged along a truss structure in a linear non-redundant array, cooled to $35 \mathrm{~K}$. A maximum baseline of $20 \mathrm{~m}$ gives a nominal resolution of 26 milli-arcseconds (mas) at $5 \mu \mathrm{m}$. Using a Fizeau (imageplane) beam combination technique, a relatively simple focal plane camera could be used to obtain both Fourier and spectral data simultaneously for a given orientation of the array. The spacecraft is rotated about the line of sight to give sufficient imaging (Fourier) data to reconstruct complex images of a broad range of astrophysical sources. Alternative and simpler three and two telescope designs emphasizing nulling and spectroscopy also were investigated and will be discussed. The culmination of the design process was a simplified two-telescope design, which was called the "C" design, and this is the design that has been studied in the greatest detail.

Several papers have been published or are in press concerning various aspects of the FKSI mission design process $[9,10]$, and in particular a very careful and thorough analysis of the detection of the extrasolar giant planets with FKSI was published recently [11]. A discussion of the requirements formulation and dynamic jitter analysis is in a companion paper in these proceedings [12].

\section{DESIGN PROCESS}

Initial design studies for the FKSI mission were performed with the Instrument Synthesis and Analysis Laboratory (ISAL) and the Integrated Mission Design Center (IMDC) at NASA's Goddard Space Flight Center. The A, B, and $\mathrm{C}$ designs were based on the preliminary ISAL and IMDC designs, but with a larger design team dedicated specifically to the FKSI mission. The goal of the process was to develop a mission that was exciting to the science team, centered around planet detection and imaging interferometry, but was practical, with a minimum of risk, and at a cost within an expected Discovery cap.

Over the course of approximately eight months our team went through three major design cycles, producing Designs A, B, and C. Costs were estimated using a grass roots approach using a NASA Work Breakdown Structure (WBS) with full cost accounting methods used for all aspects of the mission. These costs were compared with estimates using the parametric PRICE H models. In all cases costs estimated with both approaches were in reasonable agreement, roughly at the $5-10 \%$ level.

\section{DESIGN "A"}

Our initial design " $\mathrm{A}$ " with parameters displayed in Table 1 was a fully capable mission with imaging interferometry with five $1 \mathrm{~m}$ diameter afocal telescopes at $40 \mathrm{~K}$, and with a sunshade that permits a $+/-20$ degree Field-of-Regard (FOR) relative to the ecliptic (or cone half- angle relative to the anti-sun line). In addition to a science camera, which could be used for planet detection as well as general infrared astrophysics, this design also featured the option of planet detection using Bracewell nulling on two different baselines, thereby allowing planet detection simultaneously at two distinct spatial resolutions. This design covers the full 5-28 $\mu \mathrm{m}$ band available with Si:As detectors, such as are planned for the James Webb Space Telescope (JWST). This concept closely follows the community desire for a broad based TPF mission as discussed in the National Academy of Science Astronomy Decadal Survey [13], and would be an excellent pathfinder mission for TPF and its European counterpart, the Darwin mission. This concept is fully redundant in its scientific capabilities as planet detection could be done with the imaging system and imaging could be done in a limited way with the nulling subsystem. 


\begin{tabular}{|c|c|c|c|}
\hline & A - Design & B - Design & C - Design \\
\hline \multicolumn{4}{|l|}{ Key Requirements } \\
\hline Mission Life (on station) & 5 years (science operations) & 1 year (science operations) & 2 years (science operations) \\
\hline Science Capability & Imaging, Nulling & Imaging, Nulling & Nulling \\
\hline Science Wavelength Range & 5.0- 28.0 microns & $5.0-28.0$ microns & $3.0-8.0$ microns \\
\hline Science Viewing Angle & +/-20 deg off ecliptic & +/-10 deg off ecliptic & $\begin{array}{c}+/-18.5 \text { deg off ecliptic } \\
+/-7.5 \text { deg within ecliptic }\end{array}$ \\
\hline Observing Rotation Rate & $1 \mathrm{rev} / \mathrm{hr}(\max )$ & $0.5 \mathrm{rev} / \mathrm{hr}$ (max) & $2 \mathrm{rev} / \mathrm{hr}(\max )$ \\
\hline Science Data Collect Rate & 148 Gb/day (normal mode) & $61 \mathrm{~Gb} /$ day (normal mode) & $1.1 \mathrm{~Gb} /$ day (normal w/o margin) \\
\hline Instr. Thermal Stability & $1 \mathrm{~K} / \mathrm{day}$ & $1 \mathrm{~K} / \mathrm{day}$ & $1 \mathrm{~K} / \mathrm{day}$ \\
\hline Science Detector Temp & $6 K(\max )$ & 6K (max) & $35 K(\max )$ \\
\hline Near IR Detector Temps & 30K (max) & $77 \mathrm{~K}(\max )$ & $77 \mathrm{~K}(\max )$ \\
\hline Phase B Start to Launch & 54 months (no margin) & 46 months (no margin) & 61 months (incl. 6 mo. margin) \\
\hline \multicolumn{4}{|l|}{ Key Configuration Aspects } \\
\hline Science Telescopes & 5 & 3 & 2 (mounted inboard) \\
\hline Instrument Elements & AT, FT, FTS, (Nuller) & AT, FT, R=10,000 FTS, (Nuller) & $\begin{array}{c}\text { AT, FT, Nuller, } \mathbf{R}=20 \text { max } \\
\text { dispersive element }\end{array}$ \\
\hline Interferometer Type & Fizeau (image plane) & Fizeau (image plane) & Michelson (pupil plane) \\
\hline Boresight Separation & 20 m (four-fold boom) & 16 m (four-fold boom) & $12.5 \mathrm{~m}$ (two-fold boom) \\
\hline Cryocooling & $\begin{array}{l}\text { Two 6K ACTD coolers (Low } \\
\text { TRL) \& (4) } 40 \text { K coolers } \\
\end{array}$ & $\begin{array}{c}6 \mathrm{~K} \text { cryostat }\left(\mathrm{H}_{2} \text { Dewar }\right)+(2) \\
30 \mathrm{~K} \text { coolers }(\mathrm{IM} / \text { near IR detect) }\end{array}$ & $\begin{array}{c}\text { One } 32 \mathrm{~K} \text { cooler, near IR } \\
\text { detectors passively cooled }\end{array}$ \\
\hline Sunshield Location & On Bus & On Bus & On Instrument Boom \\
\hline Propulsion System & Hydrazine / He Cold Gas & All Hydrazine & All Hydrazine \\
\hline $\mathrm{ACS}$ & 6 wheel $(\mathrm{Rx}+$ momentum) & Com'l, 4 small Rx wheels & Com'l, 4 medium Rx wheels \\
\hline Star Trackers & 6 Total (4 on instr., 2 on bus) & 2 (on bus) & $\begin{array}{c}2 \text { (on bus - instr. attitude info } \\
\text { passed to bus) }\end{array}$ \\
\hline Slew Rate (peak) & $0.67 \mathrm{deg} / \mathrm{min}$ & $0.33 \mathrm{deg} / \mathrm{min}$ & Up to $6.1 \mathrm{deg} / \mathrm{min}(\mathrm{w} / 3$ wheels $)$ \\
\hline High Gain Antenna Type & 2-axis gimbal & Fixed & 2-axis gimbal \\
\hline Ground Contacts & Every 1.0 day (DSN) & Every 1.5 days (DSN) & 2x per day (com'l stations) \\
\hline Instrument Plan/Schedule & Done by Mission Ops Team & Done by Instrument team & Done by Instrument team \\
\hline Mass, Wet w/contin. (kg) & 3,710 & 3,083 & 3,183 \\
\hline Power, EOL w/contin. (W) & 1,448 & 1029 & 766 \\
\hline Fairing Diameter $(\mathbf{m})$ & 5 & 5 & 4 \\
\hline
\end{tabular}

Table 1. Trade parameter space for Designs A, B, and C. Design A allows for a complete system of 5 telescopes with both nulling planet detection and wide-field high angular resolution imaging throughout the full 5-28 micron band. Design B is an austere design, but it retains 3 telescopes and a capability for imaging but only with a single closure phase. Design C emphasizes nulling with 2 telescopes, and with restricted wavelength coverage from 3-8 microns. This design represents the "science floor" concept.

A block diagram for Design A is shown in Fig. 1, while Figs. 2 displays the basic optical design concept for the imaging system. The synthesized beam pattern (as projected onto the sky) for this design is shown in Fig. 5 of reference 9. This design has the telescopes arranged in such a way as to create "holes" in the beam pattern $\sim 0.5$ arcsec from the center of the pattern, which have sufficient depth $\left(\sim 10^{-3}\right)$ for the detection of Extra-solar Giant Planets (EGPs) as well as aperture synthesis imaging. Planet detection and synthesis imaging both require the spacecraft to rotate slowly ( $\sim$ once per hour) about the line of sight to the astronomical source.

The optical design utilizes five one meter afocal telescopes that reduce the beam by a factor of 20, as shown in Fig. 2. Next the starlight is brought to an instrument module through a series of static delays to equalize the paths to within a few millimeters, followed by a set of active delay lines that equalize pathlengths to within tens of nanometers, and then a Ritchey-Chretien beam combining telescope.

The optics and instrument are passively cooled to $35 \mathrm{~K}$ with the aforementioned sunshade, while the science detectors are cooled to approximately $8 \mathrm{~K}$. Advanced cryocoolers are used to cool the instrument assembly and the detectors. Two ancillary subsystems are required to make the science camera work, an angle tracking subsystem and a fringe tracking subsystem. The former subsystem tracks target stars at a very high precison $(<0.01$ arcsec rms $)$ and with a fast response. This system is in addition to a precision ( $\sim \mathrm{few}$ arcsec rms) tracking camera housed on the spacecraft bus. This system takes out low amplitude residual high-frequency jitter from the spacecraft bus as well as 
larger amplitude low-frequency residual pointing errors from the camera on the spacecraft bus. The fringe tracking subsystem also has a fast response and is used to stabilize the rest of the instrument from low-amplitude high-

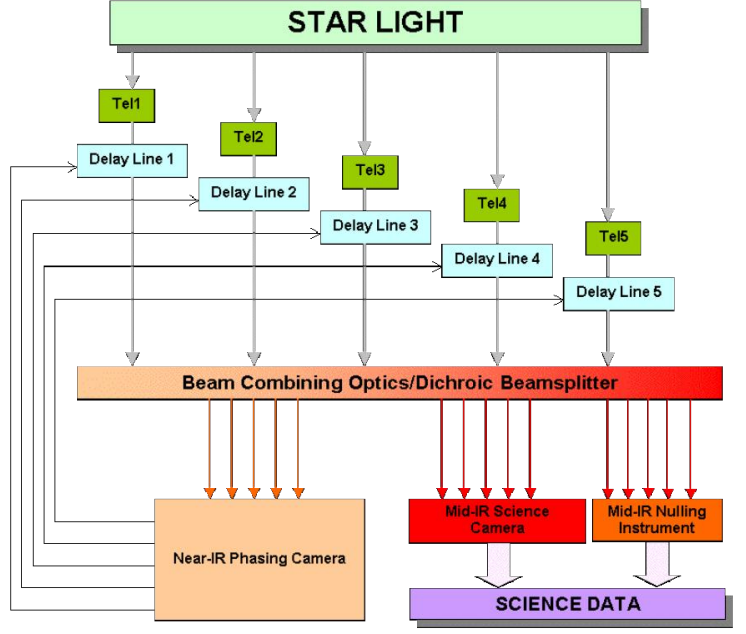

Fig. 1. Schematic diagram of Fourier-Kelvin Stellar Interferometer (FKSI) initial Design "A". This concept utilized a non-redundant linear array of 5 passively cooled telescopes. Near-infrared star light is used to phase the array, i.e., maintain zero optical pathlength difference for star light, while science data are taken with a separate mid-infrared camera and nulling instrument. frequency residual spacecraft jitter and larger amplitude low frequency delay errors from residual pointing errors.

Details of the mechanical layout are described in the figure caption. Note that the sunshield shown in Fig. 6 provides only for a $+/-20$ degree Field-of-Regard (FOR) relative to the anti-sun line. A much larger FOR, e.g., +/45 degree can be provided with a more advanced sunshade design. Note the large separation between the sunshade and the optical truss holding the telescope. This separation is an essential feature of the design and is required for the truss and telescopes to cool to $\sim 35 \mathrm{~K}$.

Other detailed features of Design "A" shown in Table I will be published elsewhere.

\section{DESIGN "B"}

Design "B" is a variant of Design "A" having three telescopes instead of five and a reduction in FOR to +/10 degrees. Other major changes include elimination of advanced cryocoolers for the detectors and replacement with a solid $\mathrm{H}_{2}$ cryostat. The detection system now uses NICMOS style detectors operating at $77 \mathrm{~K}$ for the fringe and angle tracking subsystems. The propulsion system is simplified to include only hydrazine instead of a two propellant system as in Design "A". This design retains both imaging and nulling capabilities, but the imaging is significantly reduced because of the loss of two telescopes, allowing visibility measurements on only three simultaneous baselines and a single closure phase, compared to 10 baselines and 6 closure phases for Design "A". The boom length is also reduced so that a maximum baseline of only $16.5 \mathrm{~m}$ is provided, giving a modest loss in angular resolution for the interferometer. Overall, Design "B" retains many of the essential features of Design "A" but with reduced capability and cost.

Design "B" was important to our design process as it helped clarify many of the cost and risk issues. Rather than discuss further trade-offs between Designs " $A$ " and " $B$ " we continue on to describe our "science floor" design, Design " $C$ ".

\section{DESIGN "C"}

The final design " $\mathrm{C}$ " presented here is based on a more radical reduction in scope from Design " $\mathrm{A}$ ". The mechanical layout concept for this design is shown in Fig. 4. An additional telescope is eliminated, so that only the minimum of two are provided, hence no Fizeau imaging capability is retained. Instead this design is focused on nulling detection of Extra-solar Giant Planets and on low resolution spectroscopy of their atmospheres. With

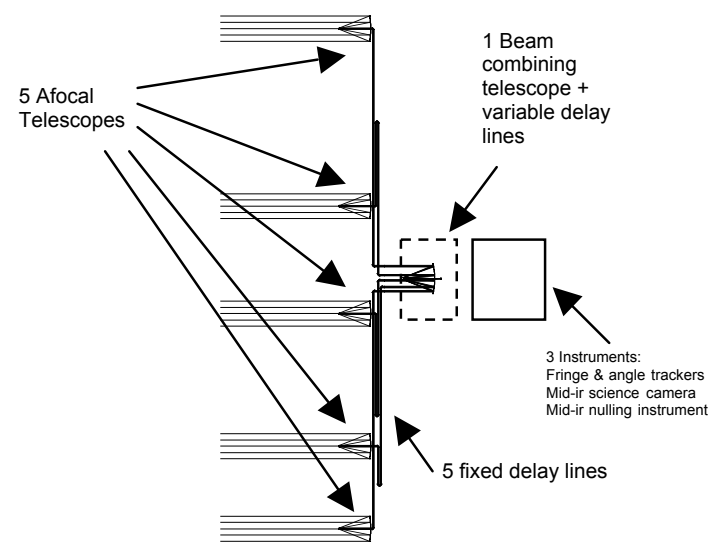

Fig. 2. Concept for Design A optical layout. In this design a wide-field of view is maintained by demagnifying the input pupil (5 afocal $1 \mathrm{~m}$ diameter telescopes on a $20 \mathrm{~m}$ maximum baseline) by a factor of 20 , and a $1 \mathrm{~m}$ Ritchey-Chretien telescope is used as a beam combiner. 

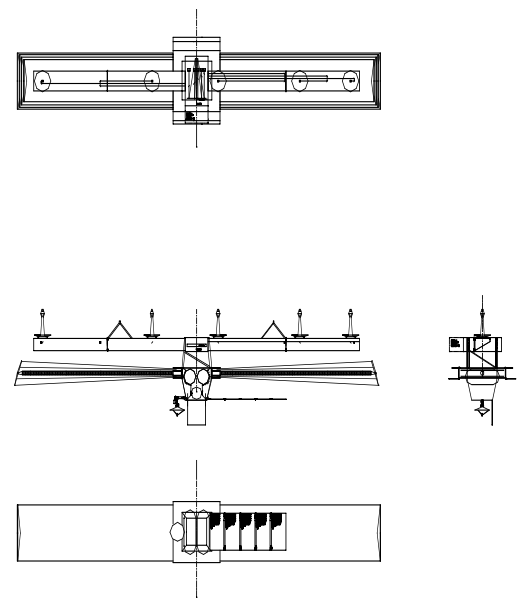

Fig. 3. Mechanical layout for Design A. Five telescopes are installed on a $20 \mathrm{~m}$ truss structure. Not shown are radiation shields around each telescope. The truss has two folds to enable the interferometer to fit into a $14 \mathrm{~m}$ long $5 \mathrm{~m}$ diameter fairing of a Delta IV launch vehicle. this reduction in scientific scope, this design is much simpler than the other two. A reduction in the operating band pass from 5-28 $\mu \mathrm{m}$ to $3-8.0 \mu \mathrm{m}$ was also chosen. This allows for a large increase in telescope and boom temperature from $35-40 \mathrm{~K}$ to $63 \mathrm{~K}$, without a decrease in sensitivity, as can be seen in Fig. 1. In addition, other major changes include reduction in telescope diameter from $1 \mathrm{~m}$ to $0.5 \mathrm{~m}$, and a change in detector technology to higher temperature $(\sim 35 \mathrm{~K})$ long-wavelength $\left(\lambda_{\text {cutoff }}=\right.$ $8.5 \mu \mathrm{m}) \mathrm{HgCdTe}$ detectors that are being developed for JWST.

The boom length is also shorter; now the baseline is 12.5 $\mathrm{m}$. The new center of the bandpass at five microns is approximately half that of the previous designs, which means the spatial resolution is approximately the same as before even with the shorter baseline. The shorter booms and the smaller telescopes in this case allow FKSI to fit into the smallest Atlas V launch vehicle, with only a $4 \mathrm{~m}$ fairing.

A cryocooler is retained, but in this case we selected a lower temperature version of the cryocooler used in the refitting of the NICMOS system at the last HST servicing mission. The cryocooler chosen is expected to contribute essentially no measurable vibrational noise to the spacecraft bus.

Design " $\mathrm{C}$ " retains a fringe and an angle tracker as in the previous designs. For a nulling interferometer, the null depth requirement $\left(10^{-4}\right)$ drives the requirements for the optical components as well as the residual pathlength jitter and pointing requirements. One important trade-off is related to the precision of the optical components versus the use of wavefront clean-up techniques, for example, by the use optical fibers, pinholes, or other techniques. A very high Strehl ratio is required to achieve an adequate null depth, and wavefront clean-up techniques are an important option, but these techniques can significantly lower the throughput of the system. Typically the throughput of an interferometer is low, on the order of a few percent, due to the large number of optical surfaces (typically 30) between the entrance aperture and the detector. Detailed studies of these issues are underway. Discussion of the complete error budget for this design including the above trades is beyond the scope of this paper and is presented in the paper by Hyde et al in these proceedings [12].

Our group has also been actively engaged in the study of detectability of EGPs based on the parameters of the " $\mathrm{C}$ " design. A numerical code in IDL has been developed to model the response of the FKSI system including contributions from exo-zodiacal dust, photon noise, pathlength jitter, read noise, and dark current noise. The modeling code uses the measured parameters for the known EGPs and simulates an observation. A preliminary result from this code is shown in Fig. 8 for 55 Cancri demonstrating the viability of planet detection with FKSI. A paper has been recently published describing some of the results of these studies [10] and a more comprehensive paper will be published later this

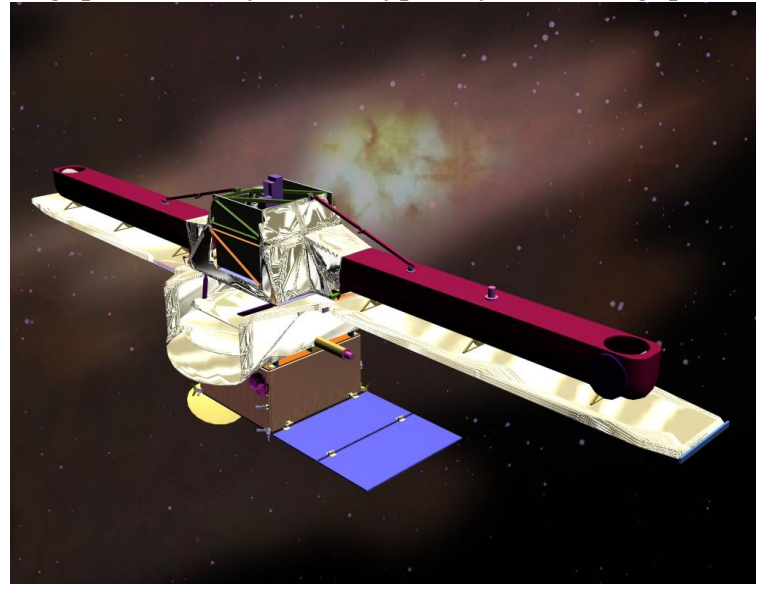

Fig. 4. Mechanical concept for FKSI Design C with system fully deployed. This design is focused on nulling interferometry for Extra-solar Giant Planet detection and low resolution spectroscopy of the atmospheres of these planets. 


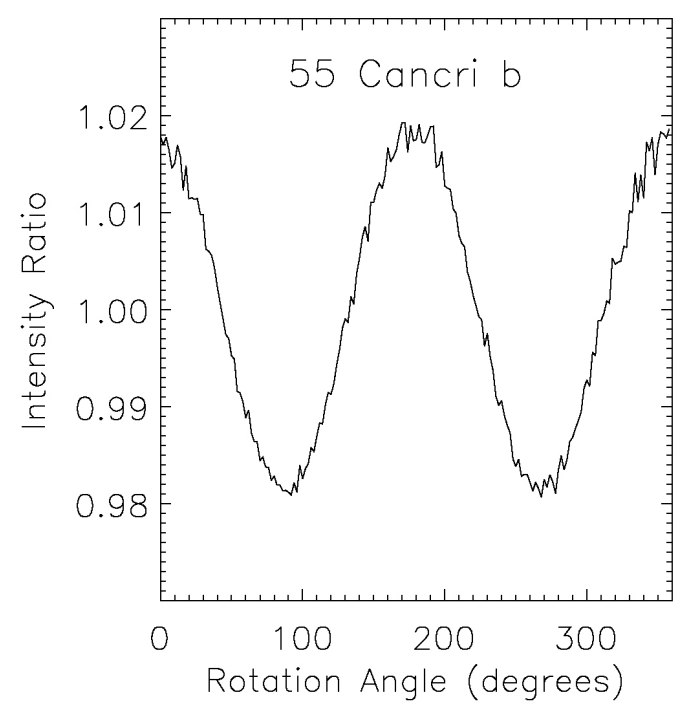

Fig. 5. Simulation of a detection of the planet around 55 Cancri (spectral type G8V) having parameters $\mathrm{Msin} i=0.84 \mathrm{M}_{\mathrm{Jupiter}}$, semi-major axis, $\mathrm{a}=0.11 \mathrm{AU}$, eccentricity, $\mathrm{e}=0.02$, and luminosity, $\mathrm{L}_{*}=0.54 \mathrm{~L}_{\text {Sun }}$. This star is at a distance of $13 \mathrm{pc}$ from the Earth. This simulation assumes a null depth of $10^{-4}$, thermal background noise, photon noise, and a residual pathlength jitter of $14 \mathrm{~nm}$ (rms). year. The most important result of the simulations has been that a large number of EGPs are detectable with the FKSI "C" design and low resolution spectroscopy can be done on the brightest of the detected planets. A broad range of ancillary science can also be done with the FKSI and a description of some other possible projects will be discussed in subsequent publications.

\section{DISCUSSION}

Detailed design studies for the FKSI mission have been performed by our group for three possible designs of decreasing scope, from one with five telescopes that allows high angular resolution imaging and nulling (Design "A"), to a mid-range design with three telescopes and imaging and nulling (Design "B"), and finally a design for nulling and spectroscopy of extra-solar giant planets (Design "C"). We have developed a very detailed understanding of costs and risks, including technical and schedule risks, but due to the confidential nature of some of these results, we have not presented them in this paper. While challenging, the FKSI mission can be achieved well within budgets like those for the new Origins Mission line.

During the next year work on the FKSI mission will be focused on completion of our testbed for the optical and control systems, which will be presented in later publications, additional more detailed simulations of the detection process for the two-telescope "C" design, and analysis of the imaging capabilities of the five telescope "A" and three telescope "B" designs. We expect to publish additional findings on the science case for a mission of this type. Given the phasing of the Terrestrial Planet Finder - Interferometer (TPF-I), which is currently scheduled for a 2019 or 2020 launch date, it makes it even more important for the US interferometry community to develop a nearer-term mission to further advance the field and develop a community of trained astrophysicists and engineers who can make the strategic mission a success. It is our hope that the FKSI mission is an appropriate stepping stone on the road to the vision of finding earth-like planets in the habitable zone of nearby stars.

\section{ACKNOWLEDGEMENTS}

Our research and development of a practical infrared imaging and nulling space interferometer has been supported by NASA Goddard Space Flight Center Internal Research and Development Funds. We thank our senior management Al Diaz, Jonathan Ormes, and Mike Ryschkewitsch for their continued support of our work. Our design team has been instrumental in the development of the mission concept, particularly team leads, George Andrew, Charles Baker, Jeff Bolognese, Rob Boyle, Gary Brown, Jason Budinoff, Julie Crooke, Bill Cutlip, Dave DiPietro, Rodger Farley, Jose Florez, Lou Hallock, Claef Hakun, Joe Howard, Tupper Hyde, Cliff Jackson, Del Jenstrom, Javier Lecha, Steve Mann, Tony Martino, Joe Pellicciotti, Tim Sauerwein, Carl Stahle, Dave Steinfeld, Lauri Via, and Mark Wilson. 


\section{REFERENCES}

[1] Marcy, G. W., Butler, R. P., Fischer, D. A., \& Vogt, S. S. 2003, in Scientific Frontiers in Research on Extrasolar Planets, ASP Conference Series vol. 294 (D. Deming \& S. Seager, eds.), p. 1

[2] Charbonneau, D., Brown, T. M., Noyes, R. W., \& Gilliland, R. L. 2002, ApJ, 568, 377

[3] Serabyn, E. 2000, in Interferometry in Optical Astronomy, SPIE Vol. 4006 (P. J. Lena \& A. Quirrenbach, eds.), p. 328

[4] Akeson, R., Swain, M. R., and Colavita, M. M., 2001, Proc. SPIE, vol. 4006, p. 321.

[5] Colavita, M. M., et al. 2003, ApJ, vol. 592L, p. 83.

[6] Beichman, C., Woolf, N. J., \& Lindensmith, C. 1999 (eds.), The Terrestrial Planet Finder (TPF): A NASA Origins Program to Search for Habitable Planets, JPL Publication 99-3.

[7] Fridlund, C. V. M., and Gondoin, P. 2003, Proc. SPIE, vol. 4852, p. 394.

[8] Lunine, J. I. et al. 2003, TPF Science Working Group, unpublished draft.

[9] Danchi, W.C., Allen, R.J., Benford, D.J., Deming, D., Gezari, D.Y., Kuchner, M., Leisawitz, D.T., Linfield, R., Millan-Gabet, R., Monnier, J.D., Mumma, M., Mundy, L.G., Noecker, C., Rajagopal, J., Seager, S., Traub, W.A., 2003, Proc. "Towards Other Earths: DARWIN/TPF and the Search for Extrasolar Terrestrial Planets," Heidelberg, Germany, 22-25 April 2003 (ESA Publication SP-539)

[10] Benford, D.J., Danchi, W.C., Allen, R.J., Deming, D., Gezari, D.Y., Kuchner, M., Leisawitz, D.T., Linfield, R., Millan-Gabet, R., Monnier, J.D., Mumma, M., Mundy, L.G., Noecker, C., Rajagopal, J., Seager, S., Traub, W.A., 2004, in the Proceedings of the October Maryland Conference.

[11] Danchi, W.C., Deming, D., Kuchner, M., \& Seager, S. 2003, ApJ, vol. 597, L57.

[12] Hyde, T.T., Liu, K-C., Blaurock, C. Bolognese, J., Howard, J.M., Danchi, W.C., Proc. SPIE, vol. 5491, these proceedings, in press, 2004.

[13] McKee, C., \& Taylor, J. (eds.) 2003, Astronomy and Astrophysics in the New Millennium 2001, National Research Council, (National Academy Press: Washington, DC) 